\title{
ON FIXED POINT THEOREMS OF NONEXPANSIVE MAPPINGS IN PRODUCT SPACES
}

\author{
KOK-KEONG TAN AND HONG-KUN XU \\ (Communicated by Palle E. T. Jorgensen)
}

\begin{abstract}
We prove some fixed point theorems for nonexpansive self- and non-self-mappings in product spaces; in particular, we provide a constructive proof of a result of Kirk and Martinez and a partial answer to a question of Khamsi. Our proofs are elementary in the sense that we do not use any universal (or ultra) nets.
\end{abstract}

\section{INTRODUCTION}

Let $X$ be a Banach space and let $C$ be a nonempty subset of $X$. We recall that $C$ is said to have the fixed point property for nonexpansive mappings (FPP for short) if every nonexpansive mapping $T: C \rightarrow C$ (i.e., $\|T x-T y\| \leq$ $\|x-y\|, x, y \in C)$ has a fixed point, and the space $X$ has FPP if every weakly compact convex subset of $X$ has FPP. For the fixed point theory of nonexpansive mappings the reader is referred to Kirk $[8,9]$ and Goebel and Reich [4].

The purpose of this paper is to prove some fixed point theorems for nonexpansive self- and non-self-mappings in product spaces. We particularly provide a constructive proof of a result of Kirk and Martinez [12] and a partial answer to a question of Khamsi [7]. We also generalize and improve upon some results of Kirk [11] and Kirk and Sternfeld [13]. Our proofs are elementary in the sense that we do not use any universal (or ultra) nets.

\section{FIXED POINT THEOREMS}

A subset $K$ of a Banach space $X$ is said to have the Browder-Göhde (B-G) property [10] if for each nonexpansive mapping $T: K \rightarrow X$, the mapping $(I-T)$ is demiclosed on $K$, i.e., for each sequence $\left\{u_{j}\right\}$ in $K$, the conditions $u_{j} \rightarrow u$ weakly and $u_{j}-T\left(u_{j}\right) \rightarrow w$ strongly imply $u \in K$ and $u-T(u)=w$. Browder [1] proved that every bounded closed convex subset of a uniformly convex Banach space has this property.

Received by the editors May 14, 1990.

1980 Mathematics Subject Classification (1985 Revision). Primary 47H10, 47H09.

Key words and phrases. Nonexpansive mapping, fixed point, weakly inward, product space. 
Now suppose $E$ and $F$ are Banach spaces with $X \subset E$ and $Y \subset F$ and let $E \oplus F$ be the product space. For $1 \leq p<\infty$ and $(x, y) \in E \oplus F$, we set

$$
\|(x, y)\|_{p}=\left(\|x\|_{E}^{p}+\|y\|_{F}^{p}\right)^{1 / p}
$$

and

$$
\|(x, y)\|_{\infty}=\max \left\{\|x\|_{E},\|y\|_{F}\right\} .
$$

It was shown in Kirk and Sternfeld [13] that if $E$ is uniformly convex (or reflexive with the B-G property), if $X$ is bounded closed convex, and if $Y$ is bounded closed and separable, then the assumption that $Y$ has the fixed point property for nonexpansive mappings assures the same is true of $(X \oplus Y)_{\infty}$. The separability assumption on $Y$ is crucial to a diagonalization process used in the proof of [13] and it is proved in [12] by using the technique of a universal (or ultra) net that the separability can be removed if the B-G property is replaced by the stronger net B-G property (the two properties are equivalent in a strictly convex Banach space [12, Remark 2]). In this section, we first show that if $X$ is weakly compact convex with the B-G property and $Y$ has the fixed point property for nonexpansive mappings, then the same is true of $(X \oplus Y)_{\infty}$. Our proof of this result is constructive. We then improve a recent result of Kirk [11] for contractive mappings. We also give a partial answer to a question of Khamsi [7]. We finally prove fixed point theorems for non-self-mappings in product spaces. Our proofs are elementary in the sense that we do not use any universal (or ultra) nets.

Theorem 2.1. Let $E$ and $F$ be Banach spaces with $X \subset E$ and $Y \subset F$. Suppose $X$ is weakly compact, convex, and has the B-G property. Suppose also $Y$ has the fixed point property for nonexpansive mappings. Then $(X \oplus Y)_{\infty}$ has the fixed point property for nonexpansive mappings.

Proof. Let $P_{1}$ and $P_{2}$ be the natural projections of $(E \oplus F)$ onto $E$ and $F$, respectively. For each fixed $y$ in $Y$, we define $T_{y}: X \rightarrow X$ by

$$
T_{y}(x)=P_{1} \circ T(x, y), \quad x \in X .
$$

Then $T_{y}$ is nonexpansive. Let $S_{y}=\left(I+T_{y}\right) / 2$ ( $I$ denotes the identity operator on $E$ ). Let $x_{0}$ in $X$ be fixed. By a result of Ishikawa [6] (see also Edelstein and O'Brien [3]), we have

$$
\lim _{n \rightarrow \infty}\left\|S_{y}\left(x_{y, n}\right)-x_{y, n}\right\|_{E}=0,
$$

where $x_{y, n}=S_{y}^{n}\left(x_{0}\right), n=1,2, \ldots$. We claim that

$$
\left\|x_{u, n}-x_{v, m}\right\|_{E} \leq\|u-v\|_{F}, \quad n, m=1,2, \ldots
$$

for $u, v$ in $Y$. Indeed, (2) is trivially valid for $n=m=1$, since

$$
\begin{aligned}
\left\|x_{u, 1}-x_{v, 1}\right\|_{E} & =\frac{1}{2}\left\|T_{u}\left(x_{0}\right)-T_{v}\left(x_{0}\right)\right\|_{E} \\
& \leq \frac{1}{2}\left\|T\left(x_{0}, u\right)-T\left(x_{0}, v\right)\right\|_{\infty} \\
& \leq \frac{1}{2}\|u-v\|_{F} .
\end{aligned}
$$


Now suppose that (2) is valid for $n, m \leq N$. Noticing

$$
\begin{aligned}
\left\|x_{u, n+1}-x_{v, m+1}\right\|_{E} & \leq \frac{1}{2}\left\|x_{u, n}-x_{v, m}\right\|_{E}+\frac{1}{2}\left\|T_{u}\left(x_{u, n}\right)-T_{v}\left(x_{v, m}\right)\right\|_{E} \\
& \leq \frac{1}{2}\left\|x_{u, n}-x_{v, m}\right\|_{E}+\frac{1}{2}\left\|T\left(x_{u, n}, u\right)-T\left(x_{v, m}, v\right)\right\|_{\infty} \\
& \leq \frac{1}{2}\left\|x_{u, n}-x_{v, m}\right\|_{E}+\frac{1}{2} \cdot \max \left\{\left\|x_{u, n}-x_{v, m}\right\|_{E},\|u-v\|_{F}\right\},
\end{aligned}
$$

we find that (2) is also valid for $n, m \leq N+1$. This verifies the validity of (2) for all $n, m \geq 1$ according to the induction principle. Now let $y(1)$ be a weak cluster point of the sequence $\left\{x_{y, n}\right\}$. Then by the B-G property of $X$ and (1), it follows that $y(1)$ is a fixed point of $S_{y}$ and hence of $T_{y}$, that is, $P_{1} \circ T(y(1), y)=y(1)$. Moreover, we have from (2) that

$$
\begin{aligned}
\|u(1)-v(1)\|_{E} & \leq \limsup _{n \rightarrow \infty}\left(\limsup _{m \rightarrow \infty}\left\|x_{u, n}-x_{v, m}\right\|_{E}\right) \\
& \leq\|u-v\|_{F} .
\end{aligned}
$$

Now let $f: Y \rightarrow Y$ be defined by

$$
f(y)=P_{2} \circ T(y(1), y), \quad y \in Y .
$$

Then for $u, v$ in $Y$, we have

$$
\begin{aligned}
\|f(u)-f(v)\|_{F} & =\left\|P_{2} \circ T(u(1), u)-P_{2} \circ T(v(1), v)\right\|_{F} \\
& \leq\|T(u(1), u)-T(v(1), v)\|_{\infty} \\
& \leq \max \left\{\|u(1)-v(1)\|_{E},\|u-v\|_{F}\right\} \\
& =\|u-v\|_{F} .
\end{aligned}
$$

Therefore $f$ is nonexpansive on $Y$ and thus has a fixed point $y \in Y$. It follows that $(y(1), y)$ is a fixed point of $T$.

Remark 2.1. After completing the present paper, the authors found that Kuczumow [14] has obtained an even stronger result than Theorem 2.1. Kuczumow's proof uses Tychonoff's theorem and a technique of Bruck [2]. However, the proof given here is constructive and elementary.

For a subset $K$ of a Banach space, a mapping $T: K \rightarrow K$ is said to be contractive if $\|T(x)-T(y)\|<\|x-y\|, x, y \in K, x \neq y$.

Theorem 2.2. Let $E$ and $F$ be Banach spaces with $X \subset E$ and $Y \subset F$. Suppose that both $X$ and $Y$ have the fixed point property for contractive mappings. Then for each $1 \leq p \leq \infty,(X \oplus Y)_{p}$ has the fixed point property for contractive mappings.

Proof. For a fixed $p, 1 \leq p \leq \infty$, suppose $T:(X \oplus Y)_{p} \rightarrow(X \oplus Y)_{p}$ is contractive. As before, for each $y$ in $Y$, we define $T_{y}: X \rightarrow X$ by

$$
T_{y}(x)=P_{1} \circ T(x, y), \quad x \in X .
$$

Then it is easily checked that in any case of $p, T_{y}$ is contractive and hence has a fixed point, i.e., there exists a point $y(1) \in X$ such that $P_{1} \circ T(y(1), y)=y(1)$. Now define $f: Y \rightarrow Y$ by

$$
f(y)=P_{2} \circ T(y(1), y), \quad y \in Y .
$$


Then for $u, v \in Y, u \neq v$, we have if $1 \leq p<\infty$,

$$
\begin{aligned}
\|f(u)-f(v)\|_{F}^{p}= & \left\|P_{2} \circ T(u(1), u)-P_{2} \circ T(v(1), v)\right\|_{F}^{p} \\
= & \|T(u(1), u)-T(v(1), v)\|_{p}^{p} \\
& -\left\|P_{1} \circ T(u(1), u)-P_{1} \circ T(v(1), v)\right\|_{E}^{p} \\
< & <(u(1), u)-(v(1), v)\left\|_{p}^{p}-\right\| u(1)-v(1) \|_{E}^{p} \\
= & \|u-v\|_{F}^{p},
\end{aligned}
$$

and if $p=\infty$,

$$
\begin{aligned}
\|f(u)-f(v)\|_{F} & =\left\|P_{2} \circ T(u(1), u)-P_{2} \circ T(v(1), v)\right\|_{F} \\
& \leq\|T(u(1), u)-T(v(1), v)\|_{\infty} \\
& <\|(u(1), u)-(v(1), v)\|_{\infty} \\
& =\max \left\{\|u(1)-v(1)\|_{E},\|u-v\|_{F}\right\} \\
& =\|u-v\|_{F},
\end{aligned}
$$

since

$$
\begin{aligned}
\|u(1)-v(1)\|_{E} & =\left\|P_{1} \circ T(u(1), u)-P_{1} \circ T(v(1), v)\right\|_{E} \\
& \leq\|T(u(1), u)-T(v(1), v)\|_{\infty} \\
& <\max \left\{\|u(1)-v(1)\|_{E},\|u-v\|_{F}\right\} \\
& =\|u-v\|_{F} .
\end{aligned}
$$

Therefore in any case of $p, f: Y \rightarrow Y$ is contractive and thus has a fixed point $y \in Y$. It follows that $(y(1), y)$ is a fixed point of $T$. The proof is complete.

Remark 2.2. Theorem 2.2 says that Theorem 2.3 of Kirk and Martinez [12] holds true for contractive mappings. It also says that the weak compactness on $X$ of Theorem 2.4 of Kirk [11] can be removed. Moreover, the technique of universal nets for contractive mappings is not necessary.

Next we consider the fixed point property for nonexpansive mappings in product spaces. Let $E$ be a Banach space. We shall say that $E$ has the property $(P)$ if for any nonconstant sequence $\left\{x_{n}\right\}$ in $E$ converging weakly to $x$, we have $\liminf _{n \rightarrow \infty}\left\|x_{n}-x\right\|<\operatorname{diam}\left(x_{n}\right)$.

Remark 2.3. It is easy to see that $E$ satisfies the property $(P)$ above if it satisfies one of the following properties:

(i) $E$ has uniformly normal structure (cf. [15]), e.g., $E$ is uniformly convex, or uniformly smooth, or $k$-uniformly rotund [17] for an integer $k>1$.

(ii) $E$ satisfies the Opial's property, that is, for any sequence $\left\{x_{n}\right\}$ of $E$, the condition $x_{n} \rightarrow x$ weakly implies $\liminf _{n \rightarrow \infty}\left\|x_{n}-x\right\|<$ $\liminf _{n \rightarrow \infty}\left\|x_{n}-y\right\|$ for $y$ in $E, y \neq x$.

(iii) The Maluta's constant $D(E)$ of $E$ (see [15]) is less than one, e.g., $E$ is nearly uniformly convex [5].

The following provides a partial answer to a question of Khamsi [7, p. 999]. 
Theorem 2.3. Let $E$ be a Banach space with the property $(P)$ above, let $F$ be a finite-dimensional space, and let $E \oplus F$ be the product space endowed with a norm satisfying the following properties:

(a) The restrictions of the norm on $E \oplus F$ to $E$ and $F$ are the initial norms of $E$ and $F$.

(b) The natural projections $P_{1}$ and $P_{2}$ associated to $E \oplus F$ have norm 1 .

Then $E \oplus F$ has the fixed point property for nonexpansive mappings.

Proof. Let $C$ be a weakly compact convex subset of $E \oplus F$ and let $T: C \rightarrow C$ be a nonexpansive mapping. Choose a subset $K$ of $C$ which is minimal with respect to being nonempty, closed, convex, and $T$-invariant. Let $\left\{z_{n}\right\}$ be a sequence in $K$ such that $\lim _{n \rightarrow \infty}\left\|T\left(z_{n}\right)-z_{n}\right\|=0$. Then by a result of Karlovitz (cf. [4]), we have

$$
\lim _{n \rightarrow \infty}\left\|z_{n}-z\right\|=\operatorname{diam}(K) \text { for all } z \text { in } K .
$$

Suppose $\operatorname{diam}(K)>0$. We may assume without loss of any generality that $\operatorname{diam}(K)=1$. Let $z_{n}=x_{n}+y_{n}$ with $x_{n} \in E$ and $y_{n} \in F$. We may also assume that $x_{n} \rightarrow x_{0}$ weakly and $y_{n} \rightarrow y_{0}$ strongly. Then $z_{n} \rightarrow z_{0}$ weakly, where $z_{0}=x_{0}+y_{0} \in K$. Noticing $\operatorname{diam}\left(x_{n}\right) \leq \operatorname{diam}\left(z_{n}\right)$ by property (b), we derive from the property $(P)$ of $E$ that

$$
\begin{aligned}
1 & =\lim _{n \rightarrow \infty}\left\|z_{n}-z_{0}\right\| \leq \liminf _{n \rightarrow \infty}\left\|x_{n}-x_{0}\right\|_{E}+\lim _{n \rightarrow \infty}\left\|y_{n}-y_{0}\right\|_{F} \\
& =\liminf _{n \rightarrow \infty}\left\|x_{n}-x_{0}\right\|_{E}<\operatorname{diam}\left(x_{n}\right) \leq \operatorname{diam}\left(z_{n}\right)=1 .
\end{aligned}
$$

This is a contradiction. Therefore, $K$ must be a singleton and the proof is complete.

Since for each $p, 1 \leq p \leq \infty$, the norm $\|\cdot\|_{p}$ satisfies the properties (a) and (b) above, we have the following:

Corollary 2.1. Let $E$ and $F$ be as in Theorem 2.3. Then the product space $E \oplus F$ with any $L^{p}$ norm for $1 \leq p \leq \infty$ has the fixed point property for nonexpansive mappings.

Finally, we prove fixed point theorems for non-self-mappings in product spaces. Recall for a closed subset $C$ of a Banach space $E$, the inward set of $C$ at an $x$ in $C, I_{C}(x)$, is defined by

$$
I_{C}(x)=\{z \in E: z=x+a(y-x) \text { for some } y \text { in } C \text { and } a \geq 0\} .
$$

A mapping $T: C \rightarrow E$ is said to be weakly inward if for each $x$ in $C, T(x)$ belongs to $\operatorname{cl} I_{C}(x)$, the closure of $I_{C}(x)$. We will say that $C$ has the fixed point property for nonexpansive weakly inward mappings if every nonexpansive weakly inward mapping $T: C \rightarrow E$ has a fixed point. If $C$ is also convex, then it is known (cf. [4]) that this property is equivalent to the fixed point property for nonexpansive mappings of $C$. 
Theorem 2.4. Let $E$ and $F$ be Banach spaces with $X \subset E$ and $Y \subset F$. Let $E \oplus F$ be the product space with an $L^{p}$ norm, $1 \leq p<\infty$. Suppose both $X$ and $Y$ have the fixed point property for nonexpansive weakly inward mappings. Then $(X \oplus Y)_{p}$ also has the fixed point property for nonexpansive weakly inward mappings.

Proof. Let $T:(X \oplus Y)_{p} \rightarrow(E \oplus F)_{p}$ be a nonexpansive and weakly inward mapping. For a fixed $y$ in $Y$, we define, as before, the mapping $T_{y}: X \rightarrow E$ by

$$
T_{y}(x)=P_{1} \circ T(x, y), \quad x \in X .
$$

Then $T_{y}$ is nonexpansive. It is also weakly inward. Indeed, for each $x \in X$, since $T(x, y) \in \operatorname{cl} I_{X \oplus Y}(x, y)$, we have

$$
T_{y}(x) \in P_{1}\left(\operatorname{cl} I_{X \oplus Y}(x, y)\right) \subseteq \operatorname{cl} I_{X}(x)
$$

as required. Hence $T_{y}$ has a fixed point $y(1) \in X$. Now define $f: Y \rightarrow F$ by

$$
f(y)=P_{2} \circ T(y(1), y), \quad y \in Y .
$$

Then it is easy to see that $f$ is nonexpansive. We now check it is also weakly inward. In fact, $f(y) \in P_{2}\left(\operatorname{cl} I_{X \oplus Y}(y(1), y)\right) \subseteq \operatorname{cl} I_{Y}(y)$. Therefore, $f$ has a fixed point $y \in Y$ and $(y(1), y)$ is a fixed point of $T$. The proof is complete.

In our final theorem, we assume $X$ has the net B-G property [12], i.e., if $T: X \rightarrow E$ is nonexpansive and if $\left\{x_{\alpha}\right\}$ is a net in $X$ for which $x_{\alpha} \rightarrow x$ weakly and $x_{\alpha}-T\left(x_{\alpha}\right) \rightarrow w$ strongly, then $x \in X$ and $x-T(x)=w$. A uniformly convex Banach space has this property.

Theorem 2.5. Let $E$ and $F$ be Banach spaces with $X \subset E$ and $Y \subset F$. Suppose $X$ is weakly compact, convex, and has the net B-G property. Suppose also $Y$ has the fixed point property for nonexpansive weakly inward mappings. Then $(X \oplus Y)_{\infty}$ has the fixed point property for nonexpansive weakly inward mappings.

Proof. Let $T:(X \oplus Y)_{\infty} \rightarrow(E \oplus F)_{\infty}$ be a nonexpansive and weakly inward mapping. Then for each $y \in Y$, the mapping $T_{y}: X \rightarrow E$ defined by

$$
T_{y}(x)=P_{1} \circ T(x, y), \quad x \in X
$$

is nonexpansive and weakly inward. Hence, the contraction $(1-t) z+t T_{y}$ is weakly inward, where $z$ is a fixed element of $X$ and $0<t<1$. Let $y(t)$ be the unique fixed point of this contraction, i.e.,

$$
y(t)=(1-t) z+t P_{1} \circ T(y(t), y) .
$$

Now by the same way as Kirk and Martinez [12, Theorem 2.3], there exists a fixed point $y(1)$ of $T_{y}$ such that

$$
\|u(1)-v(1)\|_{E} \leq\|u-v\|_{F}
$$

for all $u, v$ in $Y$. Now it is easily checked that the mapping $f: Y \rightarrow E$ defined by

$$
f(y)=P_{2} \circ T(y(1), y), \quad y \in Y
$$


is nonexpansive and weakly inward. Thus, $f$ has a fixed point $y \in Y$. It follows that $(y(1), y)$ is a fixed point of $T$. This completes the proof.

\section{ACKNOWLEDGMENT}

The authors are greatly indebted to the referee to his comments and suggestions which lead to the present form of the paper.

\section{REFERENCES}

1. F. E. Browder, Semicontractive and semiaccretive nonlinear mappings in Banach spaces, Bull. Amer. Math. Soc. 74 (1968), 660-665.

2. R. E. Bruck, Properties of fixed point sets of nonexpansive mappings in Banach spaces, Trans. Amer. Math. Soc. 179 (1973), 251-262.

3. M. Edelstein and R. C. O'Brien, Nonexpansive mappings, asymptotic regularity and successive approximations, J. London Math. Soc. 17 (1978), 547-554.

4. K. Goebel and S. Reich, Uniform convexity, hyperbolic geometry, and nonexpansive mappings, Marcel Dekker, New York, Basel, 1984.

5. R. Huff, Banach spaces which are nearly uniformly convex, Rocky Mountain J. Math. 10 (1980), 743-749.

6. S. Ishikawa, Fixed points and iteration of a nonexpansive mapping in a Banach space, Proc. Amer. Math. Soc. 59 (1976), 65-71.

7. M. A. Khamsi, On normal structure, fixed point property and contractions of type ( $\gamma$ ), Proc. Amer. Math. Soc. 106 (1989), 995-1001.

8. W. A. Kirk, Fixed point theory for nonexpansive mappings, Lecture Notes in Math., vol. 886, Springer-Verlag, 1981, pp. 484-505.

9. __ Fixed point theory for nonexpansive mappings II, Contemp. Math. 18 (1983), 121140.

10. __ Nonexpansive mappings in product spaces, set-valued mappings and $k$-uniform rotundity, Nonlinear Functional Analysis (F. E. Browder, ed.), Proc. Sympos. Pure Math., vol. 45, Part 2, Amer. Math. Soc., 1986, pp. 51-64.

11. _ An iteration process for nonexpansive mappings with applications to fixed point theory in product spaces, Proc. Amer. Math. Soc. 107 (1989), 411-415.

12. W. A. Kirk and C. Martinez Yanez, Nonexpansive and locally nonexpansive mappings in product spaces, Nonlinear Anal. 12 (1988), 719-725.

13. W. A. Kirk and Y. Sternfeld, The fixed point property for nonexpansive mappings in certain product spaces, Houston J. Math. 10 (1984), 207-214.

14. T. Kuczumow, Fixed point theorems in product spaces, Proc. Amer. Math. Soc. 108 (1990), 727-729.

15. E. Maluta, Uniformly normal structure and related coefficients, Pacific J. Math. 111 (1984), 357-369.

16. Z. Opial, Weak convergence of the sequence of successive approximations for nonexpansive mappings, Bull. Amer. Math. Soc. 73 (1967), 595-597.

17. F. Sullivan, A generalization of uniformly rotund Banach spaces, Canad. J. Math. 31 (1979), 628-636.

Department of Mathematics, Statistics and Computing Science, Dalmousie UniverSity, Halifax, Nova Scotia, CaNada B3H 3J5

Department of Mathematics, East China University of Chemical Technology, ShangHAI 200237, CHINA 